\title{
Use of Matched Filter in Direct Sequence Spread Spectrum Techniques
}

\author{
Iffat Rehman Ansari', Shahnawaz Uddin' and Sameena Naaz ${ }^{2 *}$ \\ 'University Women's Polytechnic, Aligarh Muslim University, Aligarh - 202002, Uttar Pradesh, India; \\ iffat_rehman2002@yahoo.co.in, shahnawazuddin@gmail.com \\ 2Department of Computer Science and Engineering, Faculty of Engineering and Technology, \\ Near Batra Hospital, Hamdard Nagar, New Delhi - 110062, India; \\ samy.naaz@gmail.com
}

\begin{abstract}
Objectives: The protection of signal against jamming is accomplished using Spread Spectrum (SS) techniques. In this research paper, noise rejecting property of the matched filter and Binary Phase Shift Keying (BPSK) modulation is used in Direct Sequence Spread Spectrum (DSSS) system for secure communication. Methods/Statistical Analysis: Here, DSSS system has been developed and simulated using C-programming language on windows platform. In order to perform the simulation, different parameters are initialized and message bits are generated by random function. With the help of binary message signal and PN sequence, a SS signal is generated which is modulated using BPSK modulation technique at the transmitter side. In the channel, noise/jamming signal is added to the transmitted signal. Then the received signal is demodulated using coherent BPSK receiver (i.e., correlation receiver) and matched filtering is followed by despreading the demodulated signal at the receiver side. Finally, the received binary message bits are detected by threshold detector and various parameters such as input SNR, output SNR, probability of error are calculated. Findings: It has been found that matched filter is a linear filter which has a noise rejection property (means jamming signals can be treated just like other random noise) and hence provides signal security. This particular property of matched filter is used in DSSS communication. As far as matched filter is concerned, it cannot remove the total effect of interference but still the waveforms can be detected in the presence of noise/jamming signal. So, a matched filter receiver is supposed to be an ideal receiver for a signal which is corrupted by AWGN/jamming signal. Here, an average probability of symbol error decreases because it maximizes the output signal to noise ratio. These parameters are supposed to be the most important factors that determine the efficiency of performance of the system. Applications: DSSS systems using matched filter are strong contender for mobile and personnel communications, due to their very large user handling capacity. They also have better transmission quality and capability by incorporating wireless services such as data, short transaction messages, and multimedia etc.
\end{abstract}

Keywords: Signal Security,Matched filter, DSSS, PN Sequence, SNR, Probability of Error.

\section{Introduction}

There are various methods of message security for instance, encryption, spread spectrum modulation etc. In encryption, the presence of message is not concealed, rather the information content of the message is denied to unauthenticated receivers by making various transformations. On the other hand, spread spectrum (SS) modulation is among the methods being actively used to provide protection to the transmitted signal against intentional and unintentional interference. In SS modulation, the data to be sent occupies a bandwidth in larger

${ }^{*}$ Author for correspondence 
than the minimum bandwidth necessary to send it. As the modulated signal occupies a far wider bandwidth than the bandwidth required by the original information signal, it is called spread spectrum modulation technique. The spreading of spectrum is achieved before transmission by the use of a code that does not depend on the data sequence. The receiver operates in synchronization with the transmitter and uses the same code to de-spread the received signal so that the original data may be recovered.

Spread spectrum methods have been in use for antijamming and multipath rejection applications as well as for accurate ranging and tracking. Legitimate user's communication can be effectively disrupted by jamming unless they are equipped with extremely sophisticated detection mechanisms and countermeasures. Although DSSS has an anti- jamming capability, there also exists intelligent jamming strategies that effectively attack a DSSS channel. There has been active research on developing effective jammer strategies, e.g., studied channel-oblivious and channel-aware jammers ${ }^{8}$. Spread spectrum systems are designed to work against interference of finite power. This interference could for example be due to alternate propagation paths of the signal itself, as in multipath fading, or other transmitters. In spread spectrum systems, bandwidth is sacrificed for reliability and low levels of interference with other types of communication.

Direct sequence spread spectrum communication utilizes the noise rejection property of the Matched filter. Rejection of interference, resistance against jamming, privacy of message and a number of other important properties are provided by DSSS technique ${ }^{1}$. As an example we can specify that the wide band signal cannot be jammed by a jamming signal if the spread spectrum pattern and algorithm for generating the spread sequence is not known 2 . Specifically, with the interference rejection property, commercial wireless network standards such as IEEE 802.11 and IEEE 802.15.4 widely adopt DSSS, to provide robust communications $s^{3-4}$.

Various demodulation/decoding techniques may be used for correctly reproducing the transmitted signal at the receiver end in the presence of noise but the optimum receiver for a signal corrupted by Additive White Gaussian Noise (AWGN) is the Matched filter receiver ${ }^{5}$. Matched filter receivers have also been shown to be very robust against many other forms of noise, and have thus been employed in many more applications. Not all inference will be removed by the matched filter, it can be designed such that the original signals are correctly decoded in the presence of noise. Forward Error Correction (FEC) ${ }^{6}$ is another mechanism used to improve the reliability of digital communications over wireless channels in presence of interference, fading, and noise. The median filter may also efficiently detect the original signal in presence of bipolar impulse noise $\stackrel{?}{\text {. }}$

\section{An Overview of Matched Filter}

A lot of techniques have been developed and implemented for smoothing denoising filters. Spatial domain filters can be used to reduce noise ${ }^{8}$. Some of these filters have been described in the references $\frac{9-13}{3}$. The two most promising techniques developed in the last two decades are wavelet analysis and matched filter ${ }^{14-15}$. Matched filter gives optimum signal to noise ratio (SNR) of the signal at the receiver side ${ }^{16}$. Wavelet matched filters use wavelets along with Fourier transform for recognition and classification of two dimensional objects. Phase-only Wavelet Matched Filter has been discussed in the reference ${ }^{17}$

\subsection{Matched Filter Receiver}

A linear filter with impulse response, $h_{j}(t)$, with received signal $x(t)$ used as the filter input, the resulting filter output, $y_{j}(t)$, is defined by the convolution integral:

$$
\mathrm{y}_{\mathrm{j}}(\mathrm{t})=\int_{-\infty}^{+\infty} x(\tau) \mathrm{hj}(\mathrm{t}-\tau) \mathrm{d} \tau
$$

Suppose, the impulse response is:

$$
h_{j}(t)=\emptyset_{j}(T-t)
$$

Then, the resulting filter output is:

$$
\mathrm{y}_{\mathrm{j}}(\mathrm{t})=\int_{-\infty}^{+\infty} x(\tau) \emptyset_{\mathrm{j}}(\mathrm{T}-\mathrm{t}+\tau) \mathrm{d} \tau
$$

Sampling this output at time $\mathrm{t}=\mathrm{T}$, we get:

$$
\mathrm{y}_{\mathrm{j}}(\mathrm{t})=\int_{-\infty}^{+\infty} x(\tau) \emptyset_{\mathrm{j}}(\tau) \mathrm{d} \tau
$$

Since $\phi_{\mathrm{j}}(\mathrm{T})$ is zero outside the interval $0 \leq \mathrm{t} \leq \mathrm{T}$, we finally get:

$$
\mathrm{y}_{\mathrm{j}}(\mathrm{T})=\int_{-\infty}^{+\infty} x(\tau) \emptyset_{\mathrm{j}}(\tau) \mathrm{d} \tau
$$

It is to be noted that $y_{j}(T)=x_{j}$, where $x_{j}$ is the $j^{\text {th }}$ correlator output produced by the received signal $\mathrm{x}(\mathrm{t})^{\frac{18}{18}}$. Thus the detector part of the optimum receiver may also be implemented as in Figure 1. 


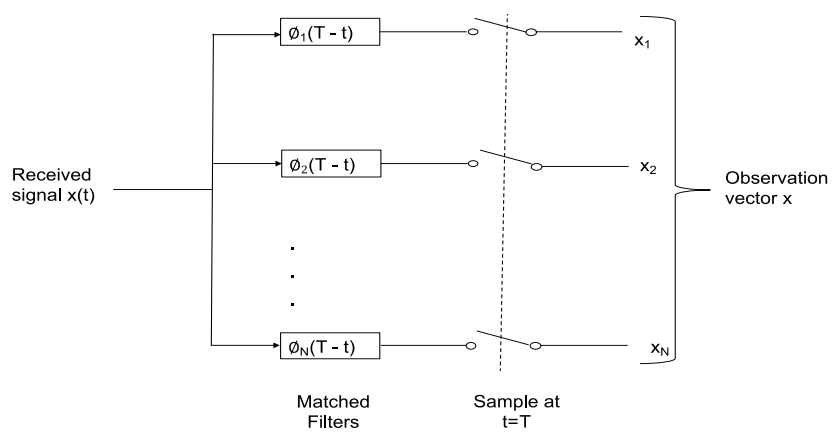

Figure 1. Detector part of matched filter receiver.

A filter is said to be matched to $\phi_{j}(t)$ if its impulse response is a delayed and time-reversed version of some signal $\phi_{j}(\mathrm{t})$, as in Eq. (2). The optimum receiver based on the detector of Figure 1 is said to be a matched filter receiver. For a matched filter operating in real time to be physically realizable, it must be causal. That is to say, its impulse response must be zero for negative time, as shown by:

$$
\mathrm{h}_{\mathrm{j}}(\mathrm{t})=0 ; \mathrm{t}<0
$$

With $h_{j}(t)$ defined in terms of $\phi_{j}(t)$ as in Eq. (2), we see that the causality condition is satisfied provided that the signal $\phi_{\mathrm{j}}(\mathrm{t})$ is zero outside the interval $0 \leq \mathrm{t} \leq \mathrm{T} \stackrel{19}{ }$.

\section{System Model}

In order to transmit the signal, the spread spectrum is carried out before phase modulation. For the purpose of analysis, however, it will be convenient to interchange the order of these two operation in the transmitter side. It is permitted to do this because the spectrum spreading and the phase shift keying are both linear operation.

Figure 2 illustrates the model of direct sequence spread spectrum PSK system. In this model, it is assumed that the effect of channel noise may be ignored because the interference limits the performance. Therefore, the output of the channel is obtained by multiplying the Pseudo Noise sequence with the Phase Shift Keyed signal and then adding the interference to it. The received signal is first multiplied by the PN sequence at the receiver, then it is fed to the coherent detector. The coherent detector input consists of a PSK signal embedded in additive code modulated interference. The modulated nature of the latter component forces the interference signal (jammer) to spread its spectrum, such that the detection of information bit at the receiver output is afforded which results in increased reliability.

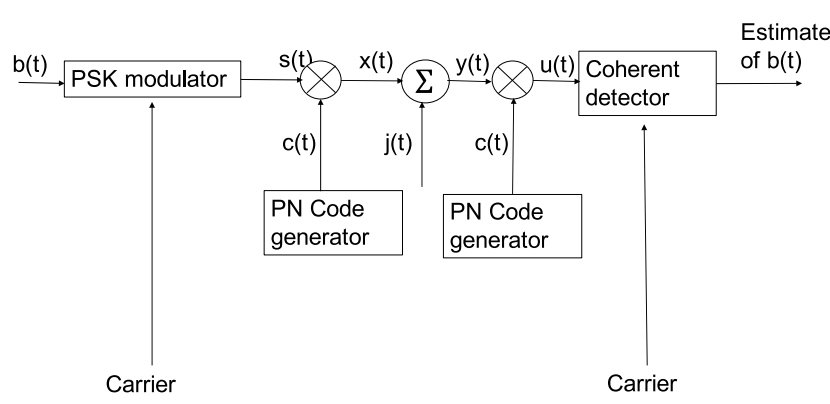

Figure 2. Model of direct sequence spread Spectrum PSK System.

\subsection{PN (Pseudo-Noise) Sequences:}

A coded sequence of 1's and 0's having certain autocorrelation properties is known as $\mathrm{PN}$ sequence. In spread spectrum communication systems, periodic PN sequences are used. PN sequences may be aperiodic and such sequences are known as Barker sequences. In order to generate the maximum length PN sequence, linear feedback shift register may be used. In a shift register having length $\mathrm{m}$, $\mathrm{m}$ flip- flops are used and they are controlled by a single timing clock and the state of each flip-flop is shifted to the next one at each pulse of the clock. The feedback function is obtained as shown in Figure 3.

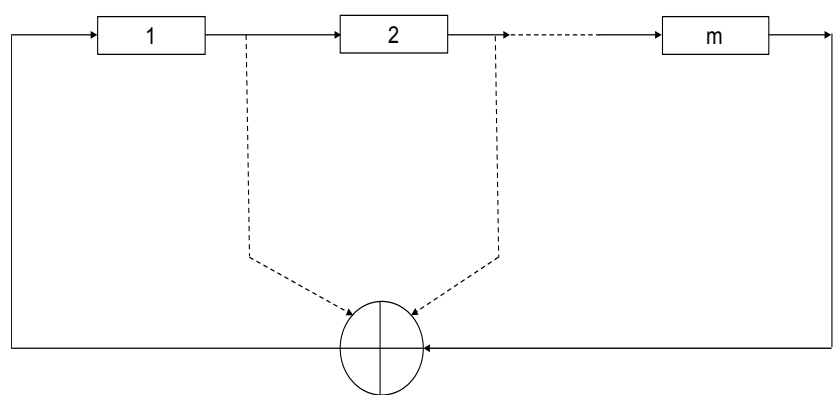

Figure 3. Maximum length sequence generator.

\section{Simulation of Matched Filter Receiver}

\subsection{Use of M-Sequence in Direct Sequence Spread Spectrum:}

1. The polar form of the data sequence $d(t)$ is assumed, i.e., the bits are represented by \pm 1 .

2. Then, the $m$-sequence produced by the feedback shift register is basically the spreading $\mathrm{PN}$-code. 
3. The error performance is simulated in the presence of one sinusoidal interfering/jamming signal having frequency of $0.49 \mathrm{kHz}$, where message sequence is produced by random number generation.

4. The error performance is again simulated in the presence of other sinusoidal interfering/jamming signal with frequencies of $0.339 \mathrm{kHz}, 0.99 \mathrm{kHz}$, and $1.34 \mathrm{kHz}$ respectively.

5. The error performance having two sinusoidal jammers of equal amplitude with the frequencies of $0.49 \mathrm{kHz}$ and $0.339 \mathrm{kHz}$ is also simulated.

6. Then, the $(\mathrm{SNR})_{\mathrm{I}}$ and $(\mathrm{SNR})_{\mathrm{O}}$ are calculated in each case.

\subsection{Block Diagram for PN Sequence Generation}

The memory shift register of $\mathrm{m}=4$ produces the $\mathrm{m}$-sequence having length of 15 . In order to generate the $\mathrm{m}$-sequence, initial states are presumed to be 1111 . This is shown in Figure 4.

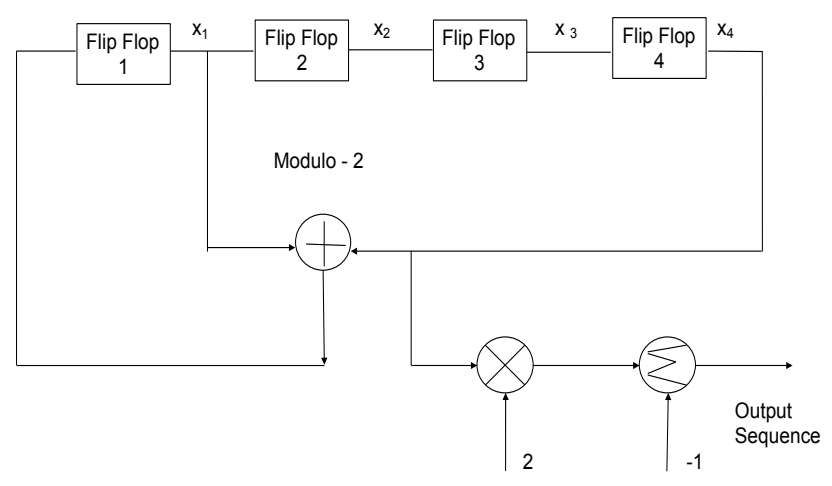

Figure 4. Feedback shift register for the m-sequence of period 15 .

The states of four flip flops are represented as $\mathrm{x}_{1}, \mathrm{x}_{2}, \mathrm{x}_{3}$ and $\mathrm{x}_{4}$ for $\mathrm{m}=4$.

The code generated is like this: $1,1,1,1,-1,1,-1,1,1$, $-1,-1,1,-1,-1,-1$

Here, $\mathrm{m}$-sequence is suited to this task because they have a broad spectrum since the runs of the same symbols are distributed.

\subsection{Direct Sequence BPSK Spread Spectrum System}

Figure 5 depicts a direct sequence spread spectrum system where an $\mathrm{m}$-sequence is used as the spreading code and BPSK as the modulation technique. The data bits are denoted by \pm 1 and feedback shift register of length $m$ $=4$ is used to produce the PN sequence. The RF carrier is modulated with the product of data sequence and PN sequence using binary phase shift keying (BPSK) at the transmitter side. An interfering/jamming signal is introduced in the channel. The BPSK demodulator is used to demodulate the received signal at the receiver side and then the output of the demodulator is multiplied by a PN sequence to nullify the effect of PN-code spreading. The coherent BPSK receiver (i.e., correlation receiver) is followed by a matched filter, the output of which is passed through a decision device where the original message signal is estimated.

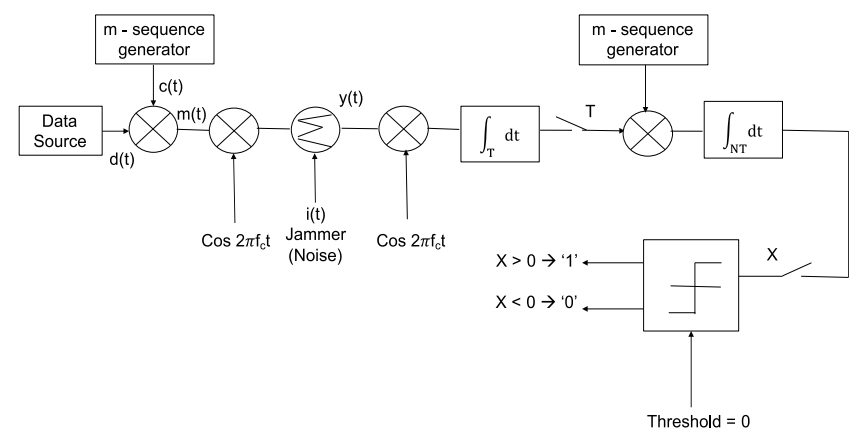

Figure 5. Direct sequence BPSK SS system.

The implementation of the simulation has been carried out in $\mathrm{C}$ language. In order to simulate a direct sequence spread spectrum system, $m$ - sequence is used as the spreading code and the value of $\mathrm{m}=4$. Here, two sinusoidal jamming signals are generated. Input and output signal to noise ratio are calculated along with the error probability and same have been displayed.

\section{Results and Discussion}

Figure 6 shows the binary form of the m-sequence, its polar waveform representation $\mathrm{c}(\mathrm{t})$, and the autocorrelation function. The auto-correlation function of $\mathrm{m}$-sequence is of periodic type, binary valued and it resembles like noise. 


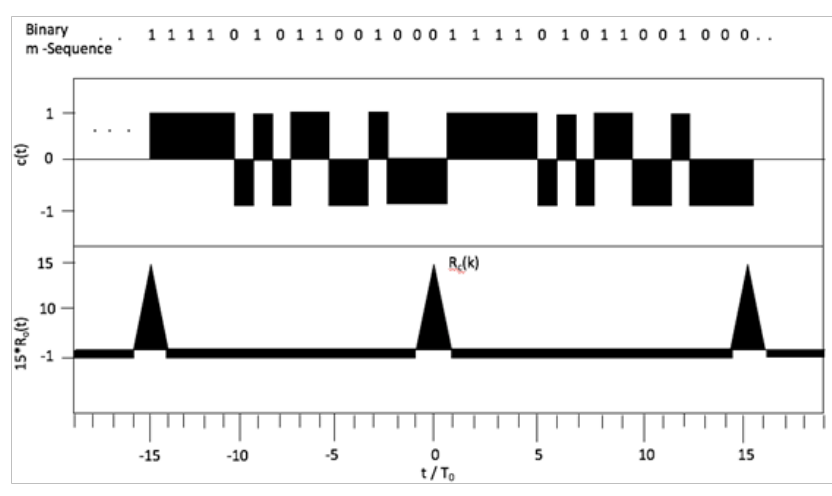

Figure 6. Polar waveform of m-sequence and autocorrelation function

Figure 7 illustrates the data sequence $d(t):[1,1,0,1,0,1$, $0,0,0,1,1,1,1,0,0]$, the modulated wave $\mathrm{m}(\mathrm{t})$, and the output of the matched filter $y(t)$. This output is same as the auto-correlation function of $\mathrm{m}$ - sequence. There are side lobes present in the filter output for an alternating sequence of 1's and 0's, and these side lobes are still $4.8 \mathrm{~dB}$ below the peaks at the appropriate sampling instant.

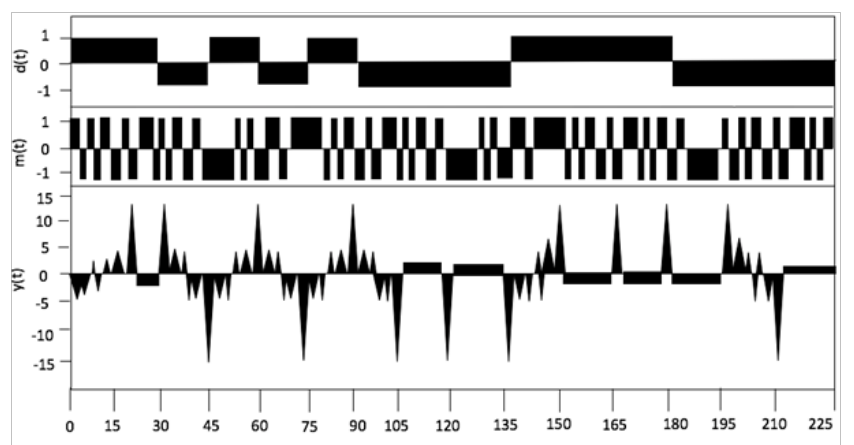

Figure 7. Modulated signal waveform and output waveform of matched filter

It is to be noted that if a DC interference is added to the modulated wave $\mathrm{m}(\mathrm{t})$, then the modulated waveform (in a bipolar format) is converted into a unipolar format having amplitude 2. This sort of the interference is the baseband equivalent of jamming a direct sequence BPSK spread spectrum system by a single tone jammer at different varying frequencies. This is not a very effective jamming method, since its only effect is to add a bias term in the matched filter output. Further, the effect of this jammer can be entirely removed by adaptively changing the threshold of the decision device.
On the other hand, if an alternating sequence of 1's and 0 's is added to the modulated m-sequence, this sort of the interference is equivalent to jamming the DS/BPSK signal with a coherent BPSK signal of the same carrier frequency. This is much more serious form of jamming because it can cause oscillations to appear in the matched filter output.

Simulation results are given in Table 1 and Table 2. Here, both input signal to noise ratio (SNR) I and output signal to noise ratio $(\mathrm{SNR})_{\mathrm{O}}$ are calculated and compared. It can be seen from the tables that an increase in the output signal to noise ratio results in decrease of probability of symbol error.

Table 1. Simulation results for a single sinusoidal jammer

\begin{tabular}{|l|c|c|c|c|}
\hline $\begin{array}{l}\text { Frequency } \\
(\mathrm{KHz})\end{array}$ & $\begin{array}{c}\text { Amplitude } \\
\text { (volts) }\end{array}$ & $\mathrm{P}_{\mathrm{e}}$ & $(\mathrm{SNR})_{\mathrm{I}}(\mathrm{dB})$ & $\begin{array}{c}(\mathrm{SNR})_{\mathrm{O}} \\
(\mathbf{d B})\end{array}$ \\
\hline 0.49 & 8 & 0.2915 & -15.05 & -1.61 \\
\hline 0.49 & 6 & 0.2061 & -12.55 & 0.88 \\
\hline 0.49 & 5 & 0.0988 & -10.97 & 2.47 \\
\hline 0.49 & 4.8 & 0.0473 & -10.61 & 2.83 \\
\hline 0.49 & 4.6 & 0 & -10.24 & 3.20 \\
\hline 0.339 & 4 & 0.2793 & -9.03 & -1.25 \\
\hline 0.339 & 3 & 0.1715 & -6.53 & 1.33 \\
\hline 0.339 & 2.5 & 0.0515 & -4.95 & 2.89 \\
\hline 0.339 & 2.4 & 0 & -4.59 & 3.23 \\
\hline 0.339 & 2.3 & 0 & -4.22 & 3.72 \\
\hline 0.99 & 8 & 0 & -15.05 & 40.34 \\
\hline 1.34 & 18 & 0.0941 & -22.10 & 2.62 \\
\hline 1.34 & 17 & 0.0133 & -21.59 & 3.10 \\
\hline 1.34 & 16.5 & 0.0049 & -21.34 & 3.36 \\
\hline 1.34 & 16.0 & 0 & -21.07 & 3.63 \\
\hline
\end{tabular}

Table 2. Simulation results for two sinusoidal jammers

\begin{tabular}{|l|c|c|c|c|}
\hline $\begin{array}{l}\text { Amplitude } \\
\text { (in volts) of } \\
\text { Jammer at } \\
\mathbf{0 . 4 5 ~ K H z}\end{array}$ & $\begin{array}{c}\text { Amplitude } \\
\text { (in volts) of } \\
\text { Jammer at } \\
\mathbf{0 . 3 3 9 ~ K H z}\end{array}$ & $\mathrm{P}_{\mathrm{e}}$ & $\begin{array}{c}(\mathrm{SNR})_{\mathrm{I}} \\
(\mathbf{d B})\end{array}$ & $\begin{array}{c}(\mathrm{SNR})_{\mathrm{O}} \\
(\mathrm{dB})\end{array}$ \\
\hline 2.0 & 2.0 & 0.0655 & -6.02 & 3.77 \\
\hline 1.8 & 1.8 & 0.0314 & -5.11 & 4.68 \\
\hline 1.7 & 1.7 & 0.0151 & -4.61 & 5.17 \\
\hline 1.65 & 1.65 & 0.0062 & -4.35 & 5.43 \\
\hline 1.62 & 1.62 & 0.0008 & -4.19 & 5.59 \\
\hline 1.60 & 1.60 & 0 & -4.08 & 5.70 \\
\hline
\end{tabular}




\subsection{Probability of Error Vs (SNR) ${ }_{0}$ for DSSS BPSK Signal}

As far as the coherent BPSK is concerned, the average probability of symbol error is defined as:

$$
\mathrm{P}_{\mathrm{e}}=1 / 2 \operatorname{erfc}\left(\mathrm{E}_{\mathrm{b}} / \mathrm{N}_{\mathrm{o}}\right)^{1 / 2}
$$

where, $\mathrm{E}_{\mathrm{b}}=$ Transmitted Bit Energy

$\mathrm{N}_{\mathrm{o}}=$ Noise power of AWGN

The complementary error function is defined as:

$$
\operatorname{erfc}(\mathrm{u})=2 /(\pi)^{2} \int \exp \left(-\mathrm{z}^{2}\right) \mathrm{dz}
$$

and we know that, $\operatorname{erfc}(\mathrm{u})= \begin{cases}1 & \mathrm{u}=0 \\ 0 & \mathrm{u}=\infty\end{cases}$

From the graph shown in Figure 8, it can be clearly seen that as the output signal to noise ratio (SNR) increases, the average probability of symbol error $\mathrm{P}_{\mathrm{e}}$ decreases. This basically explains the nature of the curve.

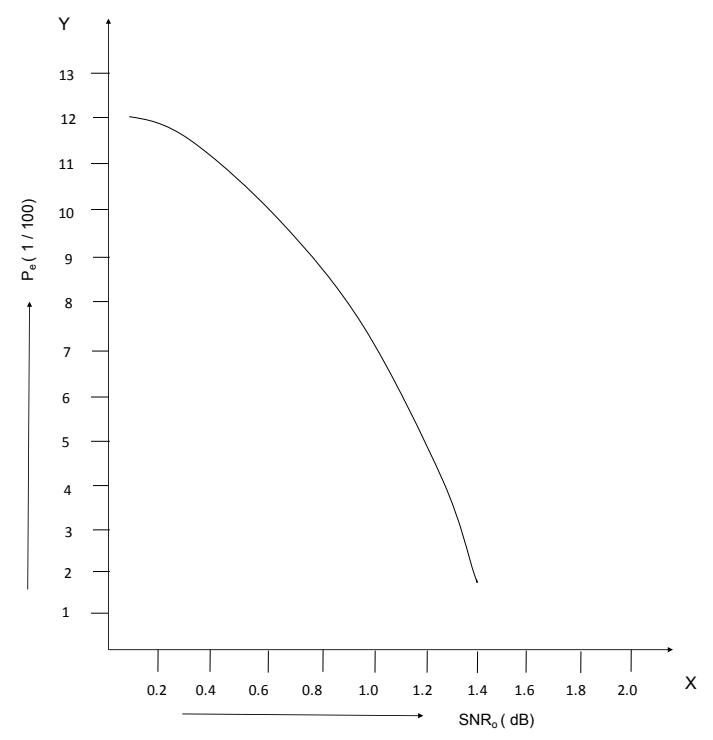

Figure 8. Graph showing Probability of error Vs $(\mathrm{SNR})_{\mathrm{O}}$ for DSSS BPSK signal

\section{Conclusion}

In this research work, DSSS system has been simulated using coherent BPSK modulation/demodulation and matched filter. Here, we discussed and explored the use of noise rejecting property of matched filter in DSSS system for signal security. From the Tables $1 \& 2$, SNRs for input signal and output signal have been compared and it is clear that an increase in the output signal to noise ratio results in the decrease of average probability of symbol error. However, this decrease in the probability of symbol error (i.e., an increase in the reliability) is achieved at the cost of system bandwidth.

\section{References}

1. Lee J S, Miller L E. CDMA Systems Engineering Handbook, Norwood, MA: Artech House Inc., 1998.

2. Simon M, Omura J, Scholtz R, Levitt B. Spread Spectrum Communications Handbook, New York, NY: McGrawHill, 1994.

3. IEEE: Institute of Electrical and Electronics Engineers, IEEE Standards for Local and Metropolitan Area Networks, Part 11: Wireless LAN Medium Access Control (MAC) and Physical Layer (PHY) Specifications, IEEE Standard 1997.

4. IEEE: Institute of Electrical and Electronics Engineers, IEEE Standard for Information Technology: Local and Metropolitan Area Networks, Specific Requirement, Part 15.4: Wireless Medium Access Control (MAC) and Physical Layer (PHY) Specifications for Low Rate Wireless Personal Area Networks (WPANs), IEEE Standard 2006.

5. Fitts correlation tracker simulation Control Lab. http:// webee.technion.ac.il/control/info/Projects/ Students/2008 /Shai Levi and NoamHelmer/Book/final_book.pdf. Date accessed: 12/04/2016.

6. Kaur N, Kalsi A P S. Implementation of Polar Codes over AWGN and Binary Symmetric Channel, Indian Journal of Science and Technology. 2016 May, 9(19), pp .1-4.

7. Kadali K S, Rajaji L, Vijayaragavan, S P. An Efficient Approach for the Removal of Bipolar Impulse Noise using Median Filter, Indian Journal of Science and Technology. 2015 Jul, 8(13) pp .1-3.

8. Kingsbury N. Complex wavelets for shift invariant analysis and filtering of signals, Journal of Applied and Computational Harmonic Analysis. 2011, 10(3), pp. 234-253.

9. Geudtner D, Schwabisch M, Winter R. SAR-interferometry with ERS-1 data, Proc. PIERS. 1994, pp. 11-15.

10. Candeias ALB, Dutra LV, Moreira JR, Mura JC. Interferogram phase noise reduction using morphological and modified median filters, Proc. IGARSS. 1995, pp. $166-168$.

11. Lee JS. Digital image enhancement and noise filtering by use of local statistics, IEEE Trans. Pattern Analysis Machine Intell. 1980, 2(2), pp. 165-168. 
12. Yu Q, Yang X, Fu S, Liu X, Sun X. An adaptive contoured window filter for interferometric synthetic aperture radar. IEEE Geoscience and Remote Sensing Letters. 2007, 4(1), pp. 23-26.

13. Meng D, Sethu V, Ambikairajah E, Ge L. A novel technique for noise reduction in InSAR images, IEEE Geoscience and Remote Sensing Letters. 2007, 4(2), pp. 226-230.

14. Grispino AS, Petracca GO, Domínguez AE. Comparative analysis of wavelet and EMD in the filtering of radar signal affected by brown noise. IEEE Latin America Transactions. 2013, 11(1), pp. 81-85.

15. Govoni MA, Hongbin LI. Range-Doppler resolution of the linear-FM noise radar waveform, IEEE Transactions on Aerospace and Electronic Systems. 2013, 49(1), pp. 658-664.
16. Islam M S, Han H, Lee J I, Jung M G, Chong U. Small target detection and noise reduction in marine radar systems, Proceedings of International Conference on Electronic Engineering and Computer Science (EECS 2013). Science Direct IERI Procedia. 2013, 4, pp. 168-173

17. Anjali M, Nelleri A. Phase-only Wavelet Matched Filtering for Two- Dimensional Image Recognition and Classification, Indian Journal of Science and Technology. 2016 Sep, 9(36), pp .1-6.

18. Signal-Space Analysis: http://www.colorado.edu/engineering/ECE/ecen4242/ch05.ppt. Date Accessed: 14/05/2016.

19. Properties of a matched filter: https://www.coursehero.com/ file/p4cn9im/Properties-of-a-Matched-Filter-We-note-thata-filter-which-is-matched-to-a/. Date Accessed: 20/5/2016. 